\title{
(C) OPEN ACCESS \\ Six ways not to improve patient flow: a qualitative study
}

\author{
Sara Adi Kreindler ${ }^{1,2}$
}

- Additional material is published online only. To view please visit the journal online (http://dx.doi.org/10.1136/ bmjqs-2016-005438).

'Community Health Sciences, University of Manitoba, Winnipeg, Manitoba, Canada ${ }^{2}$ Health Systems Performance, George and Fay Yee Centre for Healthcare Innovation, Winnipeg, Manitoba, Canada

\section{Correspondence to} Dr Sara Adi Kreindler, Community Health Sciences, University of Manitoba, 451753 McDermot Ave., Winnipeg, Manitoba, Canada R3E 0T6; skreindler@wrha.mb.ca

Received 27 February 2016 Revised 16 June 2016 Accepted 25 June 2016

Published Online First

27 July 2016
To cite: Kreindler SA. BMJ Qual Saf 2017;26:388394.

\begin{abstract}
Background Although well-established

principles exist for improving the timeliness and efficiency of care, many organisations struggle to achieve more than small-scale, localised gains. Where care processes are complex and include segments under different groups' control, the elegant solutions promised by improvement methodologies remain elusive. This study sought to identify common design flaws that limit the impact of flow initiatives.
\end{abstract}

Methods This qualitative study was conducted within an explanatory case study of a Canadian regional health system in which multitudinous flow initiatives had yielded no overall improvement in system performance. Interviews with 62 senior, middle and departmental managers, supplemented by 700 documents on flow initiatives, were analysed using the constant comparative method.

Results Findings suggested that smooth flow depends on linking a defined population to appropriate capacity by means of an efficient process; flawed initiatives reflected failure to consider one or more of these essential elements. Many initiatives focused narrowly on process, failing to consider that the intended population was poorly defined or the needed capacity inaccessible; some introduced capacity for an intended population, but offered no process to link the two. Moreover, interveners were unable to respond effectively when a bottleneck moved to another part of the system. Errors of population, capacity and process, in different combinations, generated six 'formulae for failure'.

Conclusions Typically, flawed initiatives focused on too small a segment of the patient journey to properly address the impediments to flow. The proliferation of narrowly focused initiatives, in turn, reflected a decentralised system in which responsibility for flow improvement was fragmented. Thus, initiatives' specific design flaws may have their roots in a deeper problem: the lack of a coherent systemlevel strategy.

\section{INTRODUCTION}

Ostensibly, improving patient flow should be much easier than it is. Well-established principles exist for improving timeliness and efficiency: assess capacity and demand, ascertain and address the causes of variation, streamline care processes. ${ }^{1-3}$ According to the seminal theory of constraints, the key to process improvement is identifying and addressing the major bottleneck, known as 'the constraint'; ${ }^{4}$ changes aimed at non-constraint steps cannot improve the efficiency of the process as a whole-patients may advance more quickly only to pile up at the bottleneck as badly as before. These principles have informed a variety of widely adopted flow interventions; ${ }^{5}$ they have also been packaged and repackaged into improvement methodologies (most recently lean) that organisations can employ to develop their own interventions. ${ }^{6}$ Yet the evidence on flow initiatives, be they established or bespoke, is not encouraging; a recent systematic review of interventions to reduce emergency department (ED) crowding could confirm the effectiveness of very few. ${ }^{5}$ Among ED-based interventions, even those with the strongest evidence (triage nurse ordering, triage liaison physicians, minor treatment areas (MTAs) and rapid assessment zones) have impacts that are highly variable and seldom dramatic. ${ }^{7-10}$ In the inpatient context, the family of discharge-planning and care-transition interventions shows evidence of reducing readmissions and potentially length of stay (LOS); however, in recent years, only the most complex, resource intensive of such interventions appear to yield an incremental benefit over usual care. ${ }^{11} 12$ Many flow interventions involve a net addition of resources, obscuring whether their active ingredient is efficiency improvement or merely resource enhancement. ${ }^{711}{ }^{13}$ Moreover, initiatives 
that appear to succeed in one organisation may achieve little in another, an issue that studies are only beginning to explore. ${ }^{14-16}$ Lean, for its part, has tended to fall short of expectations, typically producing only localised, small-scale gains. ${ }^{17} 18$ For most health systems, providing timely access to needed care remains a struggle. ${ }^{19}$

The 'gold standard' of the flow-improvement success story is the elegant process change; that is, one that presents a simple solution to a complex problem. ${ }^{2}$ The development of such solutions is grounded in the understanding that complex patterns of system behaviour may spring from the operation of a few simple rules and parameters, which can be identified and potentially changed. ${ }^{20}$ Indeed, in some cases, deep analysis does reveal a single problematic parameter, whose remediation transforms system functioning; ${ }^{2}{ }^{21}$ notwithstanding, there is cause to doubt whether all flow problems are truly amenable to elegant solution. A comparative case study found that lean, for example, produced substantial, sustained improvement only in departments whose care processes were relatively simple. ${ }^{16}$ In complex contexts, the offending parameter may lie outside the interveners' control, or attempts to change it may create worse problems; or, there may in fact be no single critical parameter, but rather a combination of factors that affect the outcome in non-linear and unpredictable ways. ${ }^{22}$ Even when one optimal intervention can be identified, its implementation may demand a host of facilitative interventions at other levels of the organisation. ${ }^{23}$

When analysis of a problem fails to disclose an elegant solution, decision-makers have displayed an unfortunate tendency to abandon systematic inquiry entirely, opting instead for the expedient of 'action without knowledge, 24 - or more colloquially, 'throwing a lot of spaghetti at the wall and seeing what sticks'. This response typically engenders a grab-bag of interventions that fail to resolve the problem, but compete with each other for scarce time and resources. A field that is littered with failed interventions is fertile ground for research into the causes of failure; this study, conducted within a health system in which myriad flow initiatives had yielded no discernible improvement in system performance, sought to pinpoint why interventions faltered, and by extension, what might have enabled them to succeed.

\section{METHODS}

\section{Context}

In Canada, healthcare is a provincial responsibility; most provinces have devolved its administration to regional health authorities. Serving a large city and its surrounding area, the regional health system studied ('the Region') features a matrix structure in which programmes (eg, medicine, surgery, primary care) cut across sites (hospitals and community areas). Three hospitals have become operating divisions of the Region while the other three retain their own boards; in practice, all six are accountable to the Region but enjoy considerable autonomy.

\section{Scope}

This paper reports a qualitative study undertaken within the context of an embedded explanatory case study $^{25}$ of the Region's flow-improvement efforts. The overall case study took a mixed-methods approach, incorporating (a) analysis of monthly indicators of ED and inpatient volumes and LOS 19992012; (b) review of $\sim 700$ documents on the nature, implementation and impacts of flow initiatives; (c) in-depth interviews. Parts of the case study that are not this paper's focus established several things. First, the Region showed poor flow performance; it occupied last place in comparisons with similar regions, and its ED and inpatient flow metrics gave no indication of improvement (see online supplementary appendix A). Second, it had implemented or attempted nearly every flow initiative described in the literature (see online supplementary appendix B), including interventions aimed at ED input (eg, telephone advice line, case management for frequent attenders), throughput (eg, MTAs, nurse-initiated ordering) and output (eg, short-stay unit, numerous enhancements to discharge planning). ${ }^{26}$ The outcomes of individual initiatives varied; some had demonstrably increased efficiency in some part of the care process or decreased utilisation by a certain group of patients; some had shown no benefit; some had mixed or uncertain impacts; some were unevaluated, some unevaluable. However, it can be stated that collectively, the initiatives had fallen far short of their anticipated impact on flow. The present study investigates whether any common design flaws might have played a role in this disappointing performance. (A companion study addresses system-level impediments to improvement).

In seeking out possible design flaws, it is important to distinguish between intervention failure and implementation failure. Participants described several instances in which inadequate planning and preparation, limited training, insufficient staff engagement and/or a lack of strong, in-group-based leadership prevented a project from getting off the ground. These issues are well explored in the implementation science literature, ${ }^{27} 28$ and are not specific to flow. This article is concerned with shortcomings of the intervention itself-bearing in mind, however, that intervention failure and implementation failure are sometimes intertwined (eg, an intervention's flaws may provoke staff resistance). ${ }^{24}$

\section{Methodology}

This qualitative study drew primarily on data from interviews, supplemented by document review. 
Documents (eg, evaluation reports, plans, inventories) were a major source of information on the nature and observed impacts of initiatives, and are paraphrased throughout the results section (I was not at liberty to cite or quote them directly). However, while several described barriers and facilitators to implementation, very few discussed strengths or weaknesses of intervention design.

Interview participants included 62 senior, middle and departmental managers of the Region and its programmes and sites, recruited on the basis of their role and through snowball sampling. In 1 hour semistructured interviews (see online supplementary appendix C), participants described flow initiatives in which they had been involved and elucidated what had worked well/poorly and why; they also discussed flow performance, challenges and strategies at the system level, often commenting on difficulties associated with the matrix structure. All but one participant agreed to be audiotaped, and analysis was based on verbatim transcripts. Using the constant comparative method, ${ }^{29}$ I began by open-coding hard-copy transcripts to capture all expressed opinions, experimented with various ways of organising the codes into themes using sticky notes, and settled on a working coding scheme about halfway through the transcripts. I then extracted all quotations relevant to identified themes into a Microsoft Excel spreadsheet to allow sorting and resorting. During interpretation, the idea of the constraint ${ }^{4}$ was used as a sensitising concept. ${ }^{30}$ Where appropriate, quantitative data/findings were used to verify facts or test interpretations. The analytic process continued to be iterative, moving back and forth between extracted quotations, full transcripts, documents, relevant literature and the evolving coding scheme.

\section{RESULTS}

The data illuminated three essential considerations in the design of flow initiatives: population, capacity and process. It might be postulated that smooth flow depends on linking a defined population to appropriate capacity by means of an efficient process (see figure 1). Nearly all of the many, diverse accounts of intervention failure revealed the neglect of one or more of these dimensions. The various interventions may in fact be grouped in categories according to what constellation of the three they represent, yielding 'six ways not to improve patient flow'.

\section{'Just add another form' (neither population, capacity, nor process)}

Certain initiatives apparently addressed neither population, capacity, nor process, but merely layered on new processes, positions and/or paperwork. Such endeavours were described as unsustainable at best; at worst, as invidious.
We don't ever look at what we can get rid of...the level of documentation we expect nurses to do [is] ludicrous. Little wonder they can't [help] people walk...they're tied to the pen. Because we just add another form. Every time we have a new thing, we just add another form, another piece of paper. (Manager 3)

Among the favourite targets of criticism were 'bed meetings' that, although intended to produce action on barriers to flow, allegedly produced only talk.

It's a huge chunk of every manager and program director's daily workload; they meet in the morning for huddle...then at 11:00, there's the whole regional bed call; then they go to discharge planning rounds in the afternoon... And you're lucky if you get one or two beds out of it. (Manager 27)

The majority of flawed initiatives, however, addressed one or two of the three aspects and foundered on the other(s). Most common were initiatives that focused on the 'figure' (process) but missed the 'ground' (population and/or capacity), failing to take into account that the intended population was poorly defined or the needed capacity inaccessible.

\section{Improve efficiency-in the wrong part of the process (process without capacity)}

A blinkered focus on process was evident in interventions that ameliorated the efficiency of some part of the process of care, but not the locus of greatest constraint. For example, in an effort to expedite the discharge of complex patients, one hospital piloted a complex case navigator; this initiative optimised certain steps of the process, while failing to locate and address the actual barrier to discharge.

The result was, yes we can get them ready for discharge faster-but they don't get discharged any faster. Because there's still nowhere for them to go. (Manager 30)

Initiatives developed through lean were not immune to the tendency to miss the constraint step. On the contrary, participants suggested that lean events frequently focused on too small a segment of the chain to locate the constraint, or on groups of staff with no control over it.

We get [staff] mapping out all the steps in the process and the problems-but I've not seen....a brainstorming exercise that takes a look at constraints. It's, 'Let's bring in all the links and let's see how we can optimize all the links'. I've not seen that, 'Where's the constraint?' (Manager 44)

Staff who perceived their lack of control over the constraint did not abort the lean process; rather, they knowingly focused on non-constraint steps, using lean to create workarounds that might improve patient safety in the context of what they viewed as unavoidably poor flow. One ED redesigned its processes so as 


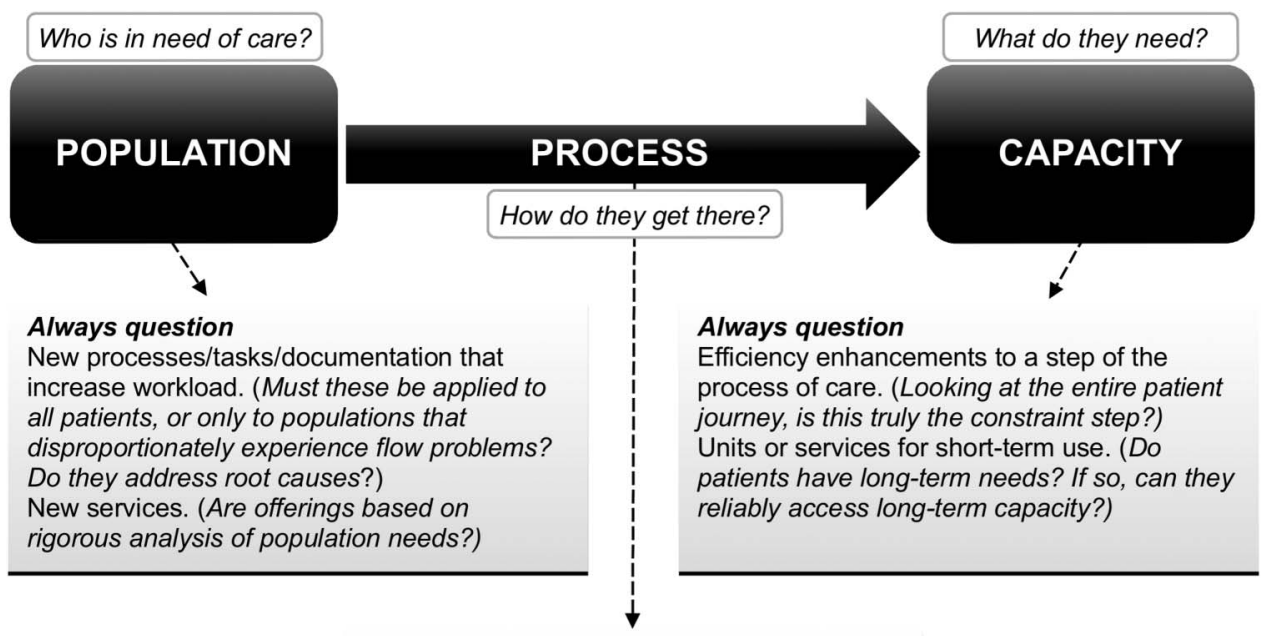

Always question
Services with multiple, unclear, or "ungated"
entry points. (How will the intended population
be connected with this service? What are the
implications if other populations use the
service?)
Processes for different populations that
converge on the same capacity. (Are some
uses of the capacity incompatible? If so, could
either set of needs be met
elsewhere/differently?)

Figure 1 Population, capacity and process: key questions for critical assessment of potential initiatives.

to give patients with chest pain quicker access to electrocardiograms, even though this would not translate into quicker access to an overcrowded department (where the constraint lay). The resulting interventions, as providers recognised, did not improve flow-and created new inefficiencies.

....it hasn't done an iota to change our flow. It's actually increased the work of the staff out front...we over-EKG everybody who has pain remotely near their hearts. (Manager 29)

\section{Create a 'parking lot' (process with capacity that does not match the duration of population needs)}

In a variation on the theme, capacity earmarked by certain initiatives for short-term use evolved into a parking lot for a population with longer-term needs but no appropriate venue. This was the reported fate of short-stay units for ED patients, transitional facilities and transition teams. The intent of such initiatives had been to stream patients away from a bottleneck; however, if a stream leads nowhere, the bottleneck is not resolved but merely relocated a short distance.

[The] problem with a lot of these quick response teams [is] you have to discharge them off. Otherwise, suddenly you've just filled up this program. (Manager 46)

\section{Design services for a poorly defined population (capacity without population)}

In a few cases, new capacity was underutilised because it turned out not to fit patient needs. In notable examples, two hospitals experimented with-but subsequently closed-subacute units. While multiple reasons were cited for each closure, a common theme was the difficulty of identifying a stable population of patients who needed subacute care and not something else. Some patients, participants reported, required rehab and belonged in the existing rehab beds; others, who did appear to require subacute care for part of their hospital stay, wound up moving back and forth between different units as their condition fluctuated, which created inefficiency and infection hazards.

We decided it really was creating more delays than it was helping, because of moving people so many times, for one thing, and a lot of energy expended on trying to find the person that was stable enough to be moved there... and quite a number of years ago we had actually established our own interim [nursing home] unit and found the same sort of thing. (Manager 25)

Data were retrieved from the most recent of these experiences; analysis showed that when the Family Medicine programme converted its subacute beds to regular beds, LOS decreased sharply, suggesting that some patients who appeared to require subacute care may no longer have belonged in hospital at all. Nonetheless, some participants continued to advocate the creation of subacute units for an ill-defined population:

There's a difficult-to-differentiate spectrum of people who need non-acute care [and are] not really good rehab candidates...they don't need our acute care 
services but it's clear that they need institutional medical care, just not any of the brands or varieties that we've actually decided to offer...so no one will take them. (Manager 21)

\section{Add capacity that is poorly targeted to its intended population (capacity without process)}

Conversely, new capacity created for a defined population sometimes fills up with patients for whom it was not intended. Managers charged with creating community-based alternatives to EDs found themselves grappling with this problem.

They said [the Crisis Response Centre is] going to decant a lot of the emergency population, but there are several problems. One is that if you open something, it meets unmet needs...And this is from community-based psychiatry, so you could get a lot of volume. (Manager 48)

Subsequent internal evaluations substantiated these concerns; after introduction of the planned community facilities, reduction in ED utilisation was barely discernible; the number of ED diversions was dwarfed by that of new users. No mechanism existed to target the new capacity to only such patients as would otherwise have visited the ED.

Even when new capacity can be formally linked to a specific population, this linkage may prove difficult to maintain. Several participants described how competing demands prevented MTAs and observation units from continuing to be used for their intended purpose.

[The MTA] was never sustainable, 'cause it was just basically part of a traditional emergency space, and so it would just always get overrun by...people who required a stretcher stuffed into that space. (Manager 35)

\section{When the bottleneck moves, do not follow it (population, capacity and process must be defined anew)}

One principle of the theory of constraints is 'the bottleneck always moves': ${ }^{4}$ once the greatest constraint is resolved, the next-greatest needs to be addressed. For continued progress, it is necessary to follow the bottleneck-but as the following example reveals, this is not always feasible.

Stakeholders throughout the Region expressed enthusiasm about the early results of the Overstay Project, a Medicine Program initiative to avert unnecessarily long hospital stays. Analysis of a comprehensive dataset had revealed that the major constraint to timely discharge was delayed decisionmaking about the disposition of complex patients, $80 \%$ of whom were discharged home after protracted indecision. Using this data, the programme developed a predictive model to identify higher-risk patients, who were subsequently screened to identify those at highest risk (population). The intervention combined changes to process-risk screening, assignment of accountability for the discharge of each high-risk patient, redirection of allied health resources away from low-risk patients-with a 'home first' policy implemented in collaboration with Home Care (capacity).

Pre/post analyses documented a significant reduction in overstay days and LOS, and participants reported that this was achieved without increasing staff workload. Yet as region-wide rollout proceeded and months went by, the predicted dramatic gains did not materialise. What happened? When contacted for follow-up, a key informant reported that the decreased LOS of patients who were discharged home was being cancelled out by a concomitant increase in LOS for patients discharged to a nursing home. The Medicine Program attempted to engage the nursing home sector in collaboratively addressing the new constraint, but made little progress. The bottleneck had moved where the programme could not followinto a sector characterised by a patchwork of forprofit and non-profit entities, most of which are outside the regional system.

\section{DISCUSSION}

The population-capacity-process triad emerged as a valuable concept for understanding the shortcomings of flow interventions. As observed, each variant of intervention failure was characterised by the neglect of one or more of its three essential components. It is important to recognise that the flawed initiatives were not arbitrary or foolish; most had some face validity, some had an evidence base, ${ }^{5}$ and even the most reviled 'add-another-form' scheme was usually modelled on a successful experience elsewhere. However, they shared a common failing: the boundaries of inquiry were too narrow to encompass the problem. $^{20}$ 'Parking lots' are a prime illustration of the pitfalls of considering only one segment of the patient journey. Transitional services may indeed be useful when they meet a need for time-limited care or reliably connect patients to other services; dysfunction occurs when the planned journey ends before patient needs are met. It is noteworthy that all the studies that have supported short-stay units investigated the delivery of protocol-driven care to a tightly defined population with short-term needs. ${ }^{13} 31$

In the mystery of the disappearing subacute patients, a focus on a single segment of the patient journey led to the attempt to design services for 'a difficult-to-differentiate spectrum of people'. 'Inpatients who do not need acute care' are not a population; this characterisation only tells us that patients are in a location where their needs are not being met; it does not describe what those needs are or illuminate why they remain unmet. Subacute care itself may indeed benefit some patients; ${ }^{32}$ the mistake lies in attempting to base service design on what patients do not need rather than on what they do. 
While an overly narrow perspective may in some cases reveal poor understanding of flow-improvement principles, in others, it may reflect interveners' lack of control over the larger system. In the Region, accountability was dispersed among programmes and sites, and many important players were outside the system altogether; often, no one had sufficient control to manage a population's entire journey across the continuum of care. In the Overstay Project, collaboration enabled two programmes to improve a patient population's journey, but their authority did not extend far enough to achieve those benefits for another population. (The implications of such fragmentation of authority will be elucidated in the companion article).

These findings help to illuminate why the 'same' intervention often produces different outcomes in different contexts. Future realist research and synthesis ${ }^{33}$ might incorporate the population-capacity-process triad as a useful frame for conceptualising the mechanisms by which, and contextual conditions under which, flow interventions exert their effects.

\section{Limitations}

This study's broad scope and retrospective nature did not permit the collection of comprehensive information about each initiative; although the triangulation of multiple data sources strengthened the ability to draw inferences, the dataset remains incomplete. Also, the outcomes of certain evidence-informed interventions (notably, nurse-initiated ordering ${ }^{10}$ ) could not be assessed due to the variability of implementation. Future research spanning multiple systems should feature parallel evaluations of similar interventions, with formal assessment of implementation and outcomes. Nonetheless, the consideration of numerous, diverse initiatives strengthened this study's potential to identify novel patterns.

The restriction of the interview sample to management-a choice made to support the pursuit of system-level issues, which managers are best situated to observe-was a significant limitation for the investigation of specific initiatives; frontline staff might have identified additional important intervention flaws, and should be included in future studies. The reliance on a single coder carried a risk of bias (although it should be noted that several participants offered detailed comments on draft reports, injecting additional perspectives into the interpretation).

As it was not possible to definitively classify each of the many initiatives as a success or failure, no formal comparison between successful and failed initiatives was undertaken. Some observations may nonetheless be made about the few initiatives that could reliably be linked to decreased LOS or utilisation. Other than the Overstay Project, these included MTAs (where maintained with discipline), services enabling specific types of patients to avoid the ED (eg, community intravenous programme), and the early 2000s shift towards day surgery. Each entailed the linkage of a defined population to new or repurposed capacity through a clear-cut process. However, as straightforward interventions are also easier to evaluate, interpretations must be drawn with caution. It should also be noted that a successful population-specific intervention may make little contribution to system-level improvement if the targeted population accounts for a low proportion of overall utilisation. Finally, one hospital did achieve a significant reduction in ED LOS and sustained this for 5 years; however, it remains difficult to determine the relative contribution of multiple interventions (some of which had additional, unintended consequences, as described in the companion article).

\section{Implications}

Figure 1 prompts decision-makers to rigorously interrogate proposed initiatives in terms of population, capacity and process. In addition, the following guiding questions provide a framework for considering the three aspects in tandem.

1. What populations are experiencing flow problems, and what are their needs? Differentiate among the major subgroups with different clusters of need; prioritise those that account for the greatest amount of potentially conservable utilisation.

2. What capacity is most suitable for meeting the identified needs, bearing in mind that different capacity may be required as patients' needs evolve? If appropriate, consider multiple suitable options, noting which ones are already present in the system.

3. What process does this population currently experience? Map the process, using data to locate the prime constraint that impedes or delays access to suitable capacity; the process' endpoint should be defined as the point at which the identified needs have been met.

4. What alternative process(es) could straightforwardly link the population to suitable capacity, avoiding the constraint? If new capacity and/or processes are proposed, how will these impact other populations? What is needed to ensure that new or released capacity is used for its intended purpose?

The suggested thought process conforms to standard flow-improvement methodology, but adds repeated reminders to take a system view, scrutinising how different parts of the system impinge on each other. Such thinking may help organisations move beyond a proliferation of piecemeal initiatives to a coherent strategy of identifying the most important constraints and following these as they move around the system. Without a system perspective to inform improvement efforts, the most promising initiative may become just another dismal entry in 'The How-Not-To Guide' to patient flow.

Acknowledgements I am grateful to the interview participants for their generosity with their time and insights, and to everyone who contributed documents and details on flow 
initiatives. I would like to extend special appreciation to those participants who provided feedback on earlier drafts; to preserve confidentiality, I am not acknowledging participants by name, but their comments contributed greatly to strengthening the analysis. My sincere thanks also go to Dr Colleen Metge for her ongoing support, guidance and review; Liping Zhang, Trevor Strome, Miroslava Svitlica and Anne Hakansson for quantitative data acquisition; and Reena Kreindler for invaluable editorial advice.

\section{Competing interests None declared.}

Ethics approval University of Manitoba Health Research Ethics Board.

Provenance and peer review Not commissioned; externally peer reviewed.

Data sharing statement For confidentiality reasons, qualitative data collected for this study cannot be shared.

Open Access This is an Open Access article distributed in accordance with the Creative Commons Attribution Non Commercial (CC BY-NC 4.0) license, which permits others to distribute, remix, adapt, build upon this work noncommercially, and license their derivative works on different terms, provided the original work is properly cited and the use is non-commercial. See: http://creativecommons.org/licenses/by$\mathrm{nc} / 4.0 /$

\section{REFERENCES}

1 Jensen K, Mayer TA, Welch SJ, et al. Leadership for smooth patient flow. Cambridge, MA: Institute for Healthcare Improvement, 2007.

2 Kreindler SA. Watching your wait: evidence-informed strategies for reducing health care wait times. Qual Manag Health Care 2008;17:128-35.

3 Showell C, Ellis L, Keen E, et al. An evidence-based review and training resource on smooth patient flow. Sandy Bay, AU: eHealth Services Research Group, University of Tasmania (on behalf of the Ministry of Health, New South Wales Government, 2012.

4 Goldratt EM, Cox J. The goal: a theory of constraints. Barrington, MA: North River Press, 1984.

5 de Grood J, Bota M, Villa-Roel C, et al. Overview of interventions to mitigate emergency department overcrowding. Review of the Quality of Care and Safety of Patients Requiring Access to Emergency Department Care and Cancer Surgery and the Role and Process of Physician Advocacy. Appendix VI. Calgary, AB: Healthcare Quality Council of Alberta, 2012:247-322. http://www.hqca.ca (accessed 30 Jun 2014).

6 Walshe K. Pseudoinnovation: the development and spread of healthcare quality improvement methodologies. Int J Qual Health Care 2009;21:153-9.

7 Abdulwahid MA, Booth A, Kuczawski M, et al. The impact of senior doctor assessment at triage on emergency department performance measures: systematic review and meta-analysis of comparative studies. Emerg Med J 2016;33:504-13.

8 Bullard MJ, Villa-Roel C, Guo X, et al. The role of a rapid assessment zone/pod on reducing overcrowding in emergency departments: a systematic review. Emerg Med J 2012;29:372-8.

9 Oredsson S, Jonsson H, Rognes J, et al. A systematic review of triage-related interventions to improve patient flow in emergency departments. Scand J Trauma Resusc Emerg Med 2011;19:43.

10 Robinson DJ. An integrative review: triage protocols and the effect on ED length of stay. J Emerg Nurs 2013;39:398-408.

11 Gonçalves-Bradley DC, Lannin NA, Clemson LM, et al. Discharge planning from hospital. Cochrane Database Syst Rev 2016;(1):CD000313.
12 Leppin AL, Gionfriddo MR, Kessler M, et al. Preventing 30-day hospital readmissions: a systematic review and meta-analysis of randomized trials. JAMA Intern Med 2014;174:1095-107.

13 Galipeau J, Pussegoda K, Stevens A, et al. Effectiveness and safety of short-stay units in the emergency department: a systematic review. Acad Emerg Med 2015;22:893-907.

14 Pearson M, Hunt H, Cooper C, et al. Providing effective and preferred care closer to home: a realist review of intermediate care. Health Soc Care Community 2015;23:577-93.

15 Pitzul KB, Lane NE, Voruganti T, et al. Role of context in care transition interventions for medically complex older adults: a realist synthesis protocol. BMJ Open 2015;5:e008686.

16 Mazzocato P, Thor J, Bäckman U, et al. Complexity complicates lean: lessons from seven emergency services. J Health Org Manage 2014;28:266-88.

17 Mazzocato P, Savage C, Brommels M, et al. Lean thinking in healthcare: a realist review of the literature. BMJ Qual Saf 2010;19:376-82.

18 Radnor ZJ, Holweg M, Waring J. Lean in healthcare: the unfilled promise? Soc Sci Med 2012;74:364-71.

19 Pines JM, Hilton JA, Weber EJ, et al. International perspectives on emergency department crowding. Acad Emerg Med 2011;18:1358-70.

20 Meadows D. Thinking in systems: a primer. White River Junction, VT: Chelsea Green, 2008.

21 Paley J. Complex adaptive systems and nursing. Nurs Inquiry 2007;14:233-42.

22 Plsek PE, Greenhalgh T. Complexity science: the challenge of complexity in health care. BMJ 2001;323:625-8.

23 Pawson R, Greenhalgh J, Brennan C, et al. Do reviews of healthcare interventions teach us how to improve healthcare systems?. Soc Sci Med 2014;114:129-37.

24 Kreindler SA. What if implementation is not the problem? Exploring the missing links between knowledge and action. Int J Health Plann Manage 2016;31:208-26.

25 Yin RK. Case study research: design and methods. 4th edn. Thousand Oaks: Sage, 2009.

26 Asplin BR, Magid DJ, Rhodes KV, et al. A conceptual model of emergency department crowding. Ann Emerg Med 2003;42:173-80.

27 Damschroder LJ, Aron DC, Keith RE, et al. Fostering implementation of health services research findings into practice: a consolidated framework for advancing implementation science. Implement Sci 2009;4:50.

28 Greenhalgh T, Robert G, Macfarlane F, et al. Diffusion of innovations in service organizations: systematic review and recommendations. Milbank Q 2004;82:581-629.

29 Strauss A, Corbin J. Basics of qualitative research: grounded theory procedures and techniques. London: Sage, 1998.

30 Charmaz K. Grounded theory: objectivist and constructivist methods. In Denzin NK, Lincoln YS, eds. Strategies for qualitative inquiry. 2nd edn. Thousand Oaks, CA: Sage, 2003:249-91.

31 Ross MA, Hockenberry JM, Mutter R, et al. Protocol-driven emergency department observation units offer savings, shorter stays, and reduced admissions. Health Aff 2013;32:2149-56.

32 Van Craen K, Braes T, Wellens N, et al. The effectiveness of inpatient geriatric evaluation and management units: a systematic review and meta-analysis. J Am Geriatr Soc 2010;58:83-92.

33 Pawson R, Greenhalgh T, Harvey G, et al. Realist review-a new method of systematic review designed for complex policy interventions. J Health Serv Res Policy 2005;10(Suppl 1):21-33. 